\title{
Vascular Atlas Formation Using a Vessel-to-Image Affine Registration Method
}

\author{
Dini Chillet ${ }^{1,2}$, Julien Jomier ${ }^{1}$, Derek Cool $^{1}$, and Stephen Aylward ${ }^{1}$ \\ 1 Computer-Aided Diagnosis and Display Lab \\ Department of Radiology, The University of North Carolina at Chapel Hill, USA \\ http://www. caddlab.rad.unc.edu \\ 2 Ecole Superieure de Chimie Physique de Lyon, 69100 Lyon, France \\ dini.chillet@cpe.fr
}

\begin{abstract}
We have developed a method for forming vascular atlases using vascular distance maps and a novel vascular model-to-image registration method. Our atlas formation process begins with MR or CT angiogram data from a set of subjects. We extract blood vessels from those data using our tubular object segmentation method. One subject's vascular network model is then chosen as a template, and its vascular distance map (DM) image is computed. Each of the remaining vascular network models is then registered with the DM template using our vascular model-to-image affine registration method. The DM images from the registered vascular models are then computed. The mean and variance images formed from those registered DM images are the vascular atlas. In this paper we apply the atlas formation process to build atlases of normal brain and liver vasculature. We use Monte Carlo simulations to demonstrate the reliability of the underlying registration method. Additionally, we explain the clinical potential of those atlases and conduct $z$-score analyses to compare individuals with the atlases to detect abnormal vessels.
\end{abstract}

\section{Introduction}

Much research has focused on generating tissue atlases. Significantly less time has been spent on forming vascular atlases. Nevertheless the interest exist, indeed if we took all of the blood vessels in the human body and laid them out in one line, the line would be approximately 100,000 miles long, and it has been shown that vessels located nearby a tumor presents "abnormal" attributes such as radius, concentration, and tortuosity [5]. Similarly, strokes may be predicted and aneurysms located using a vascular atlas. Furthermore, we hypothesize that vascular atlases might even aid in diagnosing mental disorders, such as schizophrenia, that have a strong genetic component. In particular, researchers have shown that intra-cranial vasculature actually forms prior to and potentially drives the development of the brain's tissues [10]; a vascular atlas may provide a more direct measure of the genetic component of such mental disorders.

In our previous research, a vascular model-to-image rigid registration method has been shown to be reliable and accurate [2]. In that research, the aim was 
to register the vasculature of the same patient from different scans taken at different times, e.g., MR scans before, during, or after a surgical intervention.

In this paper, we present our method for making vascular atlases using a vascular model-to-image affine registration method and a distance map (DM). A DM is an image in which each voxel's intensity corresponds to the distance to the nearest vessel. By registering an individual's vascular model (cf Fig. 1) with a DM using multi-scale intensity measures [2, we hypothesize that we can better localize corresponding functional sites despite inter-patient vascular network variability that confounds model-to-model [4] and image-to-image match metrics 86]. Thus, for a group of subjects we register each subject's vascular network with the DM (the DM is initially defined using one subject's vascular network). We then compute the DMs of those registered vascular networks; the mean and variance images of those DMs form the atlas. We present results from Monte Carlo simulations that show vascular model-to-image registration using a $\mathrm{DM}$ is reliable and therefore the resulting atlases and their measures are stable. Because of this stability our vascular atlas can be used to detect vascular anomalies within an individual and to quantify vascular network differences between populations, e.g., to detect schizophrenia.

Our method has been applied to form atlases of normal brain and liver vasculature, and basic statistical analyses have been conducted. Specifically, we computed $z$-scores to show that each atlas well represents its populations.

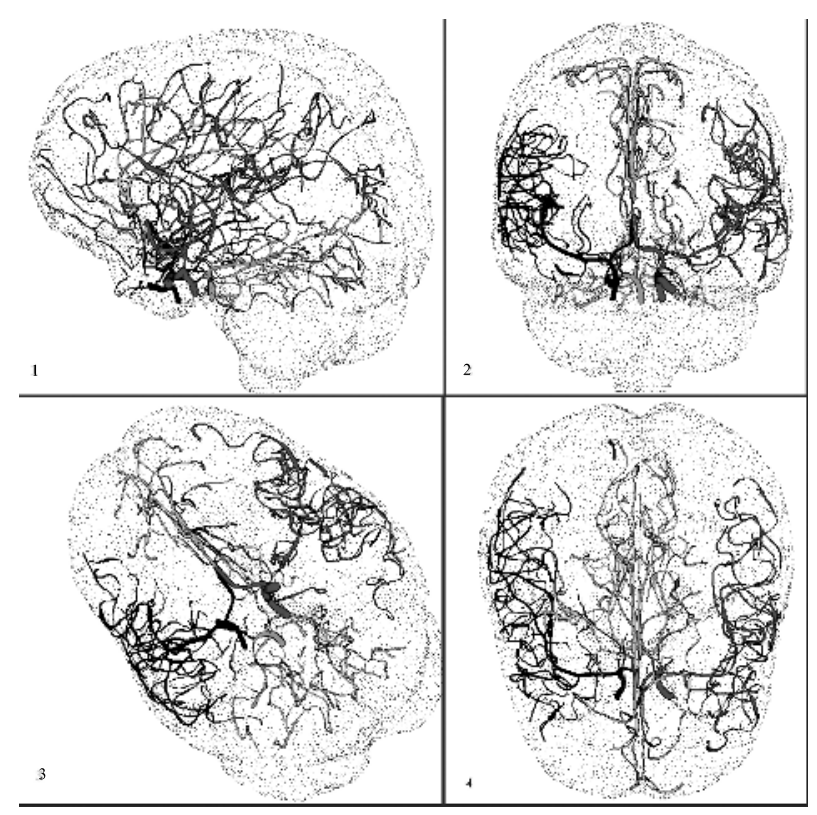

Fig. 1. Brain vasculature and tissue envelope from four different points of view: left, front, bottom, top. Data is from a normal subject's MR angiogram. Vessels were extracted using our tubular segmentation method (cf Section 2.1). 


\section{Method}

\subsection{Blood Vessels Modeling and Extraction}

The model chosen to represent a vessel is a centerline, constituted of a set of points. In our $N$-Dimensional data, each points has as components:

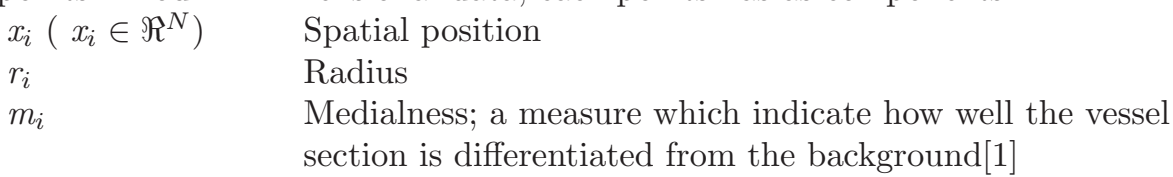

Images issued from CT or Magnetic angiogram scans can be used for our method. Blood vessels are the bright voxels in these images and are extracted using the dynamic scale intensity ridge and radius estimation method detailed in [1]. The process includes three steps: (1) selecting a seed point inside or nearby a vessel, (2) traversing the centerlines of the tubes as intensity ridges, and (3) estimating the radius at each ridge point.

\subsection{Compute Distance Map}

The computation of the DM gives an image, where the intensity value at each voxel is the distance from the closest vessel. That calculation is done using the algorithm developed by Danielsson [7]. The intensity of the DM is also inverted to get the Inverted Distance Map (IDM) in which the brightest pixels are nearest a vessel, and the intensity decrease further away from a centerline. This produces an image of "blurred vasculature." This blurriness is illustrated in Fig. 2
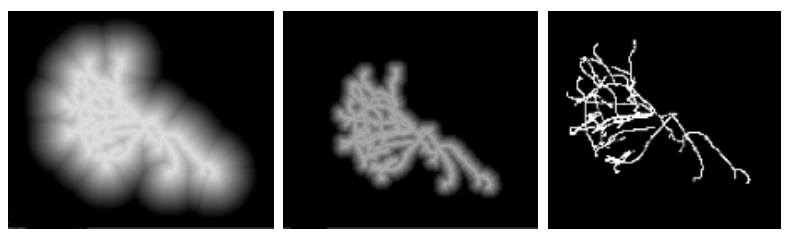

Fig. 2. Inverted Distance map(IDM) of a vasculature from the liver. The point of view is axial. On the left picture, a threshold is applied to show the intensity fall-off near the centerlines. The blurred nature of the DM vasculature network is obvious. The middle image is the same IDM, with a higher threshold; only the voxels close to a centerline point have an intensity value $\neq 0$. The right image depicts the vessel centerlines.

\subsection{Vessel-to-Image Affine Registration}

In registration the critical parts are the transform, the optimization method, and the match metric. Our atlas formation system uses an affine transform. It has a 12-dimensional parameter space. The one-plus-one evolutionary optimizer has been developed by Styner et al. [11]. It uses a normal distribution to randomly walk in parameter space to find a global maximum and updates 
the covariance matrix of the normal distribution to converge to a solution. The vessel-to-image match metric measures how well an affine matrix $\mathbf{R}$ and an offset vector o, applied to a vascular model, align that model with an image. For our system, the metric is maximal when the centerline points $x_{i}$ of the model map to the scaled brightest pixels in the IDM.

$$
F(\mathbf{R}, \mathbf{o})=\frac{1}{\sum_{j=1}^{n} w_{j}} \sum_{i=1}^{n} w_{i} \mathbf{I}_{k r_{i}}\left(\mathbf{R} x_{i}+\mathbf{o}\right)
$$

Therefore, the metric depends on the centerline sampling $\left(x_{i}\right.$ and $\left.n\right)$ and the scale $\left(k r_{i}\right)$ of the Gaussian used to blur the IDM (reducing the influence of noise [9]). Additionally, the parameter $w_{i}$ is used to weight samples depending on their radius $r_{i}$; weighting increases from $w_{i}=0$ at $r_{i}=0$ to an asymptote of $w_{i} \cong 1$ at $r_{i} \cong 3$. These parameters have been analyzed in [2] and [3].

\subsection{Atlas Formation}

The processing pipeline of the atlas formation can be divided in three steps:

First, among the $m+1$ different vascular networks $\left(V_{i}\right)$, one is chosen as a template. The chosen network's IDM is computed (cf Section 2.2) to define $I D M_{\text {template. }}$ The remaining vascular networks are then registered with $I D M_{\text {template }}$ using the vessel-to-image registration method (cf Section 2.3). Those registrations provide $m$ transformation parameter sets $\left(T_{i}\right)$, each containing a translation vector and an affine matrix.

Second, the parameters $\left(T_{i}\right)$ are applied to each $I D M_{i}$ to transform them into a common coordinate system, aligned with the template $I D M_{\text {template }}$.

Third, the mean and the variance images from the $\left(I D M_{T i}\right)$ are calculated to form the atlas. The entire pipeline is shown on Fig. 3 .

\section{Results}

Three tests were conducted using liver and brain data. These tests quantify (1) the reliability of the affine registration process for vascular model to DM registration across patients, (2) the generalization ability of the atlases to accept subjects from the same population, and (3) the ability of the atlases to discriminate subjects from different populations.

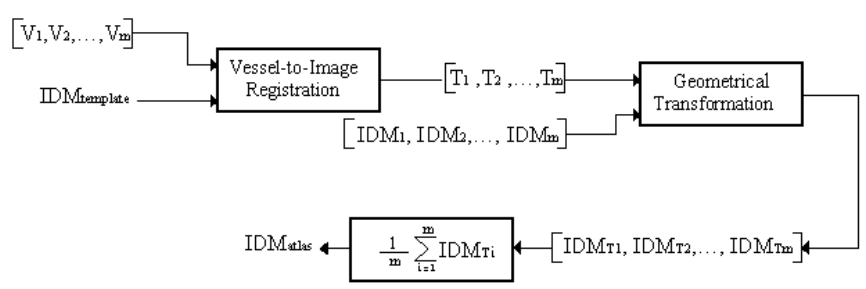

Fig. 3. Processing pipeline of the atlas formation 
Monte Carlo simulations were used to quantify the reliability of the affine registration process. We repeated 100 registrations of a vascular model from one patient's liver (cf Fig. 4) with another patient's IDM. For each registration, the vascular model was initially displaced by an offset up to \pm 10 voxels $(1 \mathrm{~cm})$ and a rotation up $\pm 0.15 \mathrm{rad}$ ( 8.5 degrees). The vascular networks of the patients were very different in terms of the number and the position of their liver vessels.

Liver vasculature is comprised of two venous systems: the portal system (shown as bright vessels in Fig. (4) brings blood into the liver; the blood is then transported out of the liver by the hepatic system (shown as dark vessels in Fig. 44). Our initial experimentation led us to separate the these vasculature networks to form two different atlases, one for the hepatic and the other for the portal system. Indeed, for some subjects, these networks were so different that it was impossible to match both hepatic and portal vessels together. Separating these systems resulted in very repeatable registrations (discussed next) and perhaps will allow the atlases to detect more subtle venous system dependent anomalies such as accessory renal veins.
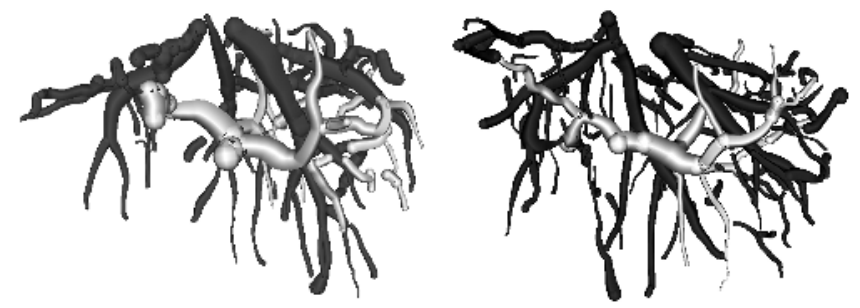

Fig. 4. Liver030(left) and Liver032(right) issued both from normal subjects. The hepatic system appears in dark and the portal in bright. These vasculatures clearly differ in vessel number and locations.

Results from the Monte Carlo simulations are given in Fig. 5 and show that only five of the one hundred instances failed to converge to a consistent solution for the hepatic system and only eight failed for the portal system. The statistics, below the two plots on the Fig. 5, indicate a low standard deviation for the offset, even for the wide range of initial mis-registrations used.

Monte Carlo simulations were also conducted using intra-cranial vascular networks from two patients; there was no need to split the vascular trees within the brain; the repeated registrations of the complete intra-cranial networks were as reliable as those of either of the venous systems in the liver.

The second analysis of the atlas focused on its ability to describe the population from which it was formed. We formed an atlas from nine MR angiograms of normal subjects and then computed the fit between those individuals and the atlas. To quantify the fit, we computed the voxel-by-voxel $z$-scores for all the subjects using the atlas' mean and variance estimates. The $z$ value indicates by how many standard deviations $\sigma$ the intensity value at a voxel of an individual's DM differs from the mean intensity value $\mu$ at that voxel as capture by the atlas.

$$
z_{i}=\left(x_{i}-\mu_{i}\right) / \sigma_{i}
$$



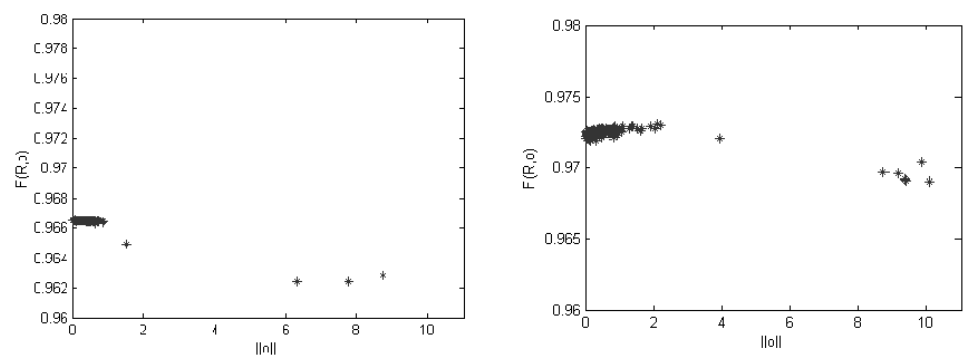

\begin{tabular}{c|ccc} 
Registration & $o_{x}$ & $o_{y}$ & $o_{z}$ \\
\hline Hepatic-Hepatic & $\mu=14.8, \sigma=0.24$ & $\mu=4.2, \sigma=0.26$ & $\mu=8.2, \sigma=0.24$ \\
Portal-Portal & $\mu=5.5, \sigma=0.46$ & $\mu=7.1, \sigma=0.48$ & $\mu=12.1, \sigma=0.47$
\end{tabular}

Fig. 5. Results of the Monte Carlo simulation for the registration of the hepatic and the portal system. The two plots show, for the 100 instances,(x-axis) the euclidean distance from the offset $\mathbf{o}$ to the average value of $\mathbf{o}$-versus- (y-axis) the metric's value. The left plot comes from Hepatic-Hepatic registration, and the right one from Portal-Portal registration. Both reveal few non-optimal registrations (on the right side of each plot) with smaller metric's value. The table shows that, after rejecting the non-optimal registrations, in both registrations the standard deviation is low which indicates consistent registrations.

With $x_{i}$ being the intensity of the voxel $i, \mu_{i}$ and $\sigma_{i}$ being respectively the atlas' mean and the standard deviation at the same position. The $z$-score calculations are limited to the volume covered by an individual's scan. Having a z-score value at each voxel allows local statistical anomalies to be detected. Calculating the histogram of $z$-scores within an individual's scan supports global comparisons across individuals as well as the verification that the population is well represented by a normal distribution.

The $z$-score distributions for the individuals that were used to form the atlas are given in Table 1 These results indicate that the atlas represents the population well, and the population has a normal distribution; however, an abnormal distribution of $z$-scores was measured in one individual. A review of that individual's anatomic and vascular data suggest a small physical deformation.

As a further test of how well the atlas represents the population, a 10th normal subject was registered with the atlas and given $z$-scores. This individual was also considered to have normal vasculature by a neurosurgeon but was not used to form the atlas. The $z$-scores for this individual are also indicated in Table 1 Those scores are well correlated with the scores from the individuals who were used to form the atlas. This indicates that the atlas does effectively represent the population.

The third validation of the atlas focused on its ability to differentiate an individual that belongs to a different population. This was tested using the MRA data from an individual in whom an arterio-venous malformation had been embolized. That data was registered with the atlas, and corresponding $z$-score values were calculated. Those results are also reported in Table 1 and illustrated 
in Fig. 6 The AVM manifested as both global and localized anomalous z-score values.

Table 1. This table show percentage of voxels, for different subjects, whom the $z$ value is under 0.4, 1 and 2 standard deviation of the atlas. AVM was a patient's images were acquired six months after surgery. Results indicate that normal subjects are well represented by the atlas, but a subject, even after treatment, is flagged.

\begin{tabular}{c|ccc} 
Percentage of voxels basing their $z$-score & $z \leq 0.4 \sigma$ & $z \leq 1 \sigma$ & $z \leq 2 \sigma$ \\
\hline Normal001 & $61 \%$ & $93 \%$ & $100 \%$ \\
Normal002 & $43 \%$ & $98 \%$ & $100 \%$ \\
Normal003 & $46 \%$ & $86 \%$ & $93 \%$ \\
Normal004 & $47 \%$ & $98 \%$ & $100 \%$ \\
Normal005 & $30 \%$ & $89 \%$ & $99 \%$ \\
Normal006 & $47 \%$ & $96 \%$ & $100 \%$ \\
Normal007 & $44 \%$ & $94 \%$ & $100 \%$ \\
Normal008 & $51 \%$ & $96 \%$ & $99 \%$ \\
Normal009 & $49 \%$ & $99 \%$ & $100 \%$ \\
\hline New Normal & $51 \%$ & $97 \%$ & $99 \%$ \\
AVM Case & $10 \%$ & $36 \%$ & $80 \%$
\end{tabular}

\section{Discussion and Conclusions}

In this paper we introduce the concept of a vascular atlas formed by registering vascular models. The basis of the registration is the calculation of a vascular distance map and the application of a multi-scale vascular model-to-image affine registration technique.

The application of our inter-patient vascular registration and atlas formation method is demonstrated using portal venous networks, hepatic venous networks, and intra-cranial vasculatures. Monte Carlo experiments quantify the reliability of the registration process for liver and intra-cranial networks. Furthermore, the intra-cranial atlas is able to correctly differentiate the vascular network from a

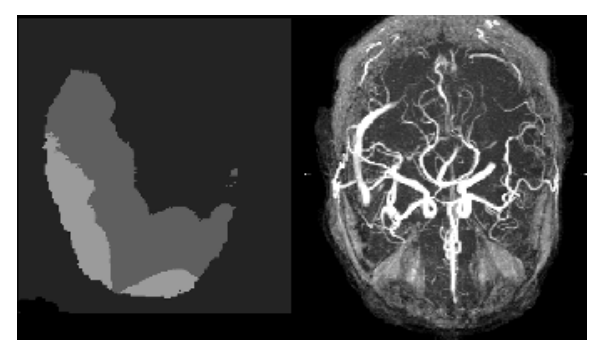

Fig. 6. Image on left shows $z$-score values $z=0, z=1$, and $z=2$ for respectively black, gray, and white voxels. These suggest an anomaly on the left side of the brain which is verified within the MR angiogram on the right 
healthy individual from a vascular network from an individual who previously had an arterio-venous malformation; the location of that lesion within the individual was also correctly identified via the atlas.

Much additional work remains to truly validate and demonstrate the utility of vascular atlases. However, this paper represents an important first step in that process. Future work will focus on the application of these atlases to identify vascular fill regions to help predict the development of collateral flow during recovery from strokes. There is also great potential in the application of the intra-cranial atlas to the early detection of mental disorders that have strong genetic components.

This project used the NLM's Insight Toolkit for Medical Image Segmentation and Registration, http://www.itk.org. This work was supported in part by the following grants: NIH/HLB R01 HL69808, Whitaker Foundation RG010341, and NLM N01 LM03501.

\section{References}

1. Aylward S, Bullit E, "Initialiation, Noise, Singularities, and Scale in Height Ridge Traversal for Tubular Object Centerline Extraction." IEEE TMI, 21(2) February 2002, pp. 61-75

2. Aylward S, Jomier J, Weeks S, Bullit E, "Registration of Vascular Images." International Journal of Computer Vision, To Appear November 2003, pages 15.

3. Aylward S, Weeks S, Bullit E, "Analysis of the Parameter Space of a Metric for Registering 3D Vascular Images." MICCAI 2001, pages 8

4. Besl PJ, McKay ND, "A method for registration of 3-d shapes," PAMI, 14(2), February 1992, pp. 239-256.

5. Bullit E, Gerig G, Pizer S, Lin W, Aylward S, "Measuring Tortuosity of the Intracerebral Vasculature from MRA Images." Accepted, IEEE-TMI, 2003.

6. Cool D, Chillet D, Kim J, Foskey M, Aylward S, "Tissue-Based Affine Registration of Brain Images to form a Vascular Density Atlas" MICCAI 2003, March 2003, Pages 8

7. Danielsson PE, "Euclidean distance mapping." Computer Graphics and Image Processing, 14, 1980, pp. 227-248.

8. Hill DLG, Batchelor PG, Holden M, Hawkes DJ, "Medical Image Registration." Phys. Med. Biol. 46, R1-R45

9. Lindeberg T, "Scale Space Theory in Computer Vision" in Kluwer Academic Publishers, Dordrecht, Netherlands, 1994

10. Seydel C "Organs Await Blood Vessels'Go Signal." Science, Vol 293 September 2001, p 2365

11. Styner M, Gerig G, "Evaluation of $2 \mathrm{D} / 3 \mathrm{D}$ bias correction with $1+1 \mathrm{ES}$ optimization," Technical Report, BIWI-TR-179 